\title{
Cómo estabilizar el nivel de empleo durante la contracción de la actividad económica. Un debate pendiente en América Latina
}

DANIEl MARTínez*

\section{INTRODUCCIÓN}

Los Fondos de estabilidad o estabilización no son hoy una novedad, al igual que tampoco lo es el establecimiento por parte de los gobiernos o los parlamentos de una "regla fiscal" que, entre otros objetivos, fija el límite del endeudamiento público. Buen número de países han aplicado políticas de ahorro fiscal (del gobierno central o de las empresas públicas) en época de crecimiento o fase alta del ciclo económico; ahorro que es "guardado" en un fondo de estabilización destinado a financiar gasto público en épocas de retracción económica y baja tasa de captación de ingresos fiscales. En la mayoría de los casos el fondo de recursos ahorrados se destina, cuando ocurre la retracción económica, a financiar políticas sociales públicas, en especial de protección social, con la finalidad de que la pobreza no aumente a niveles socialmente insoportables, o se destina a proteger determinadas actividades sectoriales cuando se produce un caída del precio internacional de los productos generados en esos sectores (commodities) o un alza del precio de productos importados que son utilizados como insumos industriales (petróleo y celulosa básicamente). Se trata principalmente, en este último caso, de Fondos de Estabilización de precios en la industria energética (Uruguay es un caso bastante conocido), en la minería (Chile es un ejemplo de ello con la estabilización de los precios del cobre), en la industria forestal o en otras industrias exportadoras como las agrícolas y las ganaderas (Argentina creó un fondo de estabilización financiado con "retenciones" a los ingresos por exportaciones agropecuarias).

En este artículo se pasa revista a diferentes modalidades de intervención existentes para estabilizar el nivel de empleo y/o asegurar un ingreso mínimo a las personas o familias, y se plantea además la alternativa de una nueva modalidad (los fondos de estabilización del empleo) que podría ser sumamente eficaz para el mantenimiento del nivel de empleo en períodos de contracción de la actividad económica ${ }^{1}$.

* Es miembro de la "Red Euro-Latinoamericana de Análisis sobre Trabajo y Sindicalismo" (www.RELATS.org) y de la Asociación "Compromiso Asturias XXI".

1 Deseo expresar mi agradecimiento a María Noel Hernández, Ricardo Infante y Jaime Mezzera, quienes leyeron el borrador de este artículo y me hicieron muy valiosos comentarios. 


\section{EL CICLO ECONÓMICO Y EL EMPLEO}

Cuando la recesión o fase baja del ciclo económico se presenta, una de las primeras cosas que resultan afectadas es el nivel de empleo, sobre todo del empleo formal. Con la retracción económica siempre aumenta el desempleo y el peso del empleo informal y del trabajo precario. Resultado de ello, aumenta también la pobreza, la desigualdad distributiva y la inequidad social.

Los procesos de transmisión de los efectos de la contracción de la actividad económica sobre el empleo y los ingresos son de sobra conocidos y explicados en múltiples investigaciones de la CEPAL, OIT, de la OCDE y del Banco Mundial, por citar algunas de las más importantes ${ }^{2}$, por lo que no es necesario detenerse mucho en ellos en este artículo.

Cuando se inicia un período de contracción económica se produce un triple fenómeno en el mercado de trabajo: 1) el aumento del desempleo; 2) el aumento de la informalidad y el trabajo precario; 3) la caída del salario real medio.

La caída del salario real se debe al hecho de que la oferta de trabajo (alimentada por los nuevos cesantes) supera largamente la demanda del mismo por parte de las empresas. Es decir, la fila de los que hacen cola para pedir trabajo en la puerta de las empresas aumenta, pero la puerta no se abre para la mayoría de ellos.

Los cesantes como consecuencia de la contracción de la actividad económica pueden ir al desempleo, pero en los países en los que no existe seguro de desempleo o este es muy débil, buena parte de los cesantes no pueden darse el lujo de quedar sin ingresos y optan por auto emplearse como informales o por aceptar trabajos "en negro", sin protección social y con salarios extremadamente bajos. El resultado es un aumento de la informalidad y de la precariedad laboral. Otra parte, sin embargo, opta, voluntaria o involuntariamente (en este último caso, especialmente mujeres y jóvenes), por quedar cesante viviendo bien del seguro de desempleo (si puede) bien de las redes de protección familiar.

Ahora bien, además de este aumento del desempleo como consecuencia de la destrucción de empleos, también puede haber un aumento adicional de la desocupación debido al aumento de la tasa de participación. Aunque puede parecer contradictorio, en muchos países y en ocasiones cuando se inicia un periodo recesivo y ante el aumento de los miembros de la familia en condición de cesantes (en general mayormente jóvenes y mujeres), otros miembros de la familia hasta entonces inactivos pueden salir a buscar trabajo para compensar la caída del ingreso familiar, con el consiguiente aumento de la tasa de participación. Si esos nuevos "buscadores de trabajo" no consiguen un empleo pasan a alimentar el desempleo.

2 CEPAL. Weller, J. Crecimiento, empleo y distribución del ingreso en América Latina. Serie Macroeconomía del Desarrollo $\mathrm{n}^{\circ} 22,2012$. OIT. Informe sobre el empleo en el mundo. Diversos ańos. OCDE. Employment Outlook. Diversos años. Banco Mundial. Informe sobre Desarrollo Mundial 2013 Empleo. 
Cuando se supera el periodo recesivo y se vuelve a una nueva fase alta del ciclo económico, se produce un aumento del nivel de ocupación, pero es un aumento más lento y con un cierto rezago (uno o dos semestres como mínimo) respecto del crecimiento de la actividad económica. Sin embargo, los últimos beneficiados de la recuperación del empleo son las mujeres y los jóvenes (los que fueron los primeros en ser despedidos) ya que las empresas priorizan la contratación de varones de mediana edad, anteriormente asalariados y con mayor experiencia técnico profesional.

Este aumento de la tasa de ocupación al recuperarse la actividad económica pude ocasionar una reducción de la tasa de desempleo o no, dependiendo de cómo responda la tasa de participación. Usualmente, cuando se reactiva la economía la tasa de participación baja y, por tanto, el desempleo baja aún más debido a que muchas personas que se habían incorporado al mercado de trabajo al producirse la recesión económica, se retiran al no requerir ya seguir trabajando porque otros miembros de la familia han vuelto a trabajar. Sin embargo, si la reactivación económica es fuerte y prolongada puede ocurrir que no solo nadie se retire del mercado de trabajo sino que muchos desempleados desalentados (desempleo oculto) opten por buscar una nueva oportunidad laboral. En ese caso, el desempleo podría incluso aumentar a pesar de la reactivación de la economía.

Para superar esta situación de destrucción de empleo, aumento de la informalidad y del desempleo y caída del salario real medio no hay otra solución que generar empleo de buena calidad, ya que estos mecanismos de trasmisión de los efectos de la retracción económica hacia el empleo y, con ello, a los niveles de pobreza no son correas de transmisión que se pueden "romper" con solo gasto público para financiar políticas sociales (principalmente transferencias hacia los sectores sociales vulnerables). Si no hay generación de empleo suficiente y de buena calidad, la política de transferencias (condicionadas o no) no basta para reducir la pobreza por sí sola. Como muchos economistas han señalado reiteradamente, la mejoría de la situación de mercado de trabajo reduce la pobreza y la desigualdad en enteros, mientras que el aumento del gasto social la reduce en solo décimas. Es por ello que en situaciones de contracción de la actividad económica con destrucción de empleo, las intervenciones de los gobiernos y del sector privado para retomar la generación de empleo es crucial, y el debate sobre cuáles de esas modalidades de intervención son eficientes o no se torna un debate relevante y necesario.

\section{MODALIDADES DE INTERVENCIÓN PARA MANTENER EL NIVEL DE EMPLEO Y/O PARA GENERAR INGRESOS}

$\mathrm{Si}$, como he señalado en párrafos anteriores, cuando la actividad económica se contrae también se produce una caída del nivel de empleo, sobre todo formal, entonces cabe preguntarse cómo intervienen los gobiernos y el sector privado para hacer frente a esta situación. 
La pregunta tiene, además, una especial actualidad ahora que han sido aprobados por la Asamblea General de las Naciones Unidas los objetivos de desarrollo sostenible a alcanzar antes el año 2030, uno de cuyos objetivos, el número 8, es promover el crecimiento económico sostenido, inclusivo y sostenible, el empleo pleno y productivo y el trabajo decente para todos ${ }^{3}$.

A este objetivo general en materia de empleo se vinculan los cuatro objetivos específicos siguientes:

- Promover políticas orientadas al desarrollo que apoyen las actividades productivas, la creación de empleo decente, el emprendimiento, la creatividad y la innovación, y alentar la oficialización y el crecimiento de las microempresas y las pequeñas y medianas empresas, entre otras cosas mediante el acceso a servicios financieros.

- Para 2030, lograr el empleo pleno y productivo y garantizar un trabajo decente para todos los hombres y mujeres, incluidos los jóvenes y las personas con discapacidad, y la igualdad de remuneración por trabajo de igual valor.

- Para 2020, reducir sustancialmente la proporción de jóvenes que no están empleados y no cursan estudios ni reciben capacitación.

- Para 2020, desarrollar y poner en marcha una estrategia mundial para el empleo de los jóvenes y aplicar el Pacto Mundial para el Empleo de la Organización Internacional del Trabajo.

En los párrafos que siguen describo sintéticamente algunas de las más conocidas iniciativas y modalidades de intervención gubernamental para generar ingresos o para mantener el nivel de empleo en los diferentes países del mundo pero en especial en Europa y América Latina; iniciativas de intervención que, sin embargo, en algunos casos no han logrado aún pasar del nivel de propuesta o de aplicación a modo de prueba.

Una primera propuesta es la de "trabajo garantizado" (TG). Se trata de una propuesta desarrollada por economistas del Centro para el Pleno Empleo y la Igualdad (Centre of Full Employment and Equity - CofFEE) de la Universidad de Newcastle de Australia y de la Kansas City School of Economists y el Centro para el Pleno Empleo y la Estabilidad de Precios, estos dos últimos de la Universidad de Missouri-Kansas City ${ }^{4}$.

La propuesta de TG consiste en garantizar que el Estado ofrezca un trabajo con salario fijo a cualquier persona que desee trabajar. Para disponer de esos trabajos el gobierno tiene que mantener siempre una reserva de puestos de trabajos disponibles. Esta reserva debe ser mayor cuando el empleo en el sector privado disminuye y menor cuando el empleo en este sector aumenta. Es decir, el fondo de estabilización de puestos de trabajo disponible tendría una dinámica similar a la de los actuales seguros de desempleo.

3 www.un.org/sustainabledevelopment/es/objetivos-de-desarrollo-sostenible/

4 Ver El trabajo garantizado. Una propuesta necesaria frente al desempleo y la precarización. Guzmán, A., Trillo F. y otros. Madrid: Editorial Akal, 2015. 
Al mantenerse constante la cantidad de empleos del país (por la absorción por el Estado del empleo destruido por el sector privado), la demanda privada proveniente de los ingresos de los trabajadores sería relativamente estable y, por ello, el impacto inflacionario del TG sería muy escaso o incluso nulo. A su vez, el salario fijo que se le otorga al trabajador beneficiado por este sistema actuaría como salario mínimo de la economía.

Hasta ahora, en ningún país se ha aplicado una política de TG pero algunos sí se han puesto en marcha programas de empleo con algunas similitudes con las que tendría la política de TG5. Es el caso de "Jefes y Jefas de Hogar" de Argentina (2002); el Servicio de empleo de la Commonwealth en Australia (1940-1970); los Programas de Inversión Social (FIS) en diversos países de América Latina; el programa mínimo de trabajo en programas de obras públicas en Chile (1975-1987); el Plan maestro contra el desempleo en Corea (1997-1998); los Programas de trabajo público en el marco del New Deal (WPA, PWA, CWA) en los Estados Unidos de América (1933-1936); el Esquema Maharashtra de Garantía de Empleo en la India (192-2005); el PAIT en Perú (19852000); el programa de expansión de trabajos públicos de Sudáfrica (2004 en adelante); el programa "socialización de la inversión" de Suecia (1938-1970), entre otros.

En los Estados Unidos existe una ley (Humphrey-Hawkins) que autoriza al gobierno a crear una reserva de empleos públicos para garantizar empleo a trabajadores que no encuentran ocupación (o que la pierden) en el sector privado. Sin embargo, esa reserva de empleos nunca fue constituida.

Otra propuesta de intervención también amplia, en términos de cobertura, es la de la renta básica universal planteada en 1995 por Van Parijs y otros intelectuales europeos, y que tiene muchos seguidores en los diferentes continentes ${ }^{6}$. Esta renta básica se define como el derecho de todo ciudadano o residente en un país a percibir periódicamente del Estado una cantidad de dinero que le sirva para, cuando menos, atender las necesidades básicas sin que se le exija a cambio contraprestación alguna. Esta renta, al ser universal, podría otorgársele a cada persona desde el momento de su nacimiento y el sistema sería financiado por el régimen general de impuestos que el Estado recauda de la ciudadanía.

En el caso de quienes ya perciben un ingreso, el sistema de renta básica estaría asociado al régimen de impuesto a la renta o ingresos de cada persona, entendiendo por ello que actuaría como un impuesto negativo (un descuento impositivo o desgravamen parcial) que variaría en función de los ingresos adicionales de cada ciudadano.

La propuesta, si bien ha tenido y tiene muchos impulsores, especialmente desde círculos cercanos a posiciones políticas y económicas que priorizan los objetivos de una justa distribución de la riqueza y de una mayor equidad social, también ha generado un gran rechazo, en especial desde sectores políticos y económicos vinculados al liberalismo,

\footnotetext{
5 Ver Eduardo Garzón Espinosa, «Experiencias internacionales de empleo garantizado», La Marea. Noviembre, 2014.

6 Ver publicaciones sobre renta básica universal en: www.basicincome.org
} 
que consideran que una renta de este tipo desincentiva el esfuerzo personal y, por tanto, los aumentos sostenidos de productividad y de competitividad, lo que afecta las posibilidades de progreso. Es por ello que hasta ahora la aplicación de un régimen de renta básica universal solo se aplica, y como prueba, en Finlandia, Irlanda y Holanda, y su aplicación iba a ser sometida a referéndum en Suiza al momento de escribir este artículo.

Otro mecanismo aplicado, pero que no debe de ser confundido con el de la renta básica universal, es el de la renta minima de inserción o salario social. Se trata de una ayuda a quienes están en situación de pobreza, o en riesgo de caer en ella, y que no disponen de los ingresos mínimos necesarios para satisfacer sus necesidades básicas (alimentación, vivienda, transporte, especialmente). Habitualmente es una prestación de naturaleza económica, compuesta de un monto fijo y otro variable en función de la cantidad de miembros de la familia. Sin embargo, en algunas ocasiones y en algunos países, la contribución también puede ser en especie (sobre todo alimentos) o en exoneraciones de pago, total o parcial, de determinados servicios públicos como luz y agua.

Bajo diferentes denominaciones, este sistema de renta mínima de inserción opera en muchos países del mundo. En América Latina opera en Argentina ("Prestaciones familiares" desde 1953 y "Asignación universal por hijo" desde 2009), en Bolivia ("Prestaciones familiares" desde 1956, "Bono Juancito Pinto" desde 2008), en Brasil ("Salario familiar" desde 1963 y "Programa de garantía de renta mínima” desde 1991), en Colombia ("Asignaciones familiares" 1957), en Costa Rica ("Asignaciones familiares" desde 1975) y en Uruguay ("Asignaciones familiares" desde 1943), por citar solo algunos casos.

Además de este tipo de modalidades de intervención orientadas a ayudar y/o proteger bien a todas las personas sin excepción, bien a colectivos sociales en situación de pobreza o en riesgo de caer en ella, existen otros mecanismos de atención de carácter individual y que se orientan a proteger al trabajador que ya está desempleado (solo el cesante, no el desempleado que busca trabajo por primera vez) o que podría llegar a estarlo en algún momento.

Se trata, por una parte, de los seguros de desempleo ${ }^{8}$, financiados con aportes tanto del trabajador como del empleador o, como el caso del Uruguay, con recursos fiscales, y destinados a otorgar una prestación económica durante un cierto tiempo (seis o más meses) a quien ha perdido su empleo. Actualmente se exige cada vez más que para percibir dicha prestación la persona desempleada busque activamente un empleo y/o participe en actividades de capacitación y formación profesional.

\footnotetext{
Ver Rojo Torrecilla, E. y García Nieto, J., Lucha contra la pobreza y cambio social: renta minima garantizada y salario ciudadano. Madrid: Cáritas Española, 1989. Godoy, Lorena, Programas de renta minima vinculada a la educación: las becas escolares en Brasil. Santiago de Chile: Naciones Unidas, CEPAL, 2014.

8 CEPAL, Seguros de desempleo, objetivos, características y situación en América Latina, 2003.
} 
Según información de la Oficina Internacional de Trabajo,

[...] de los 198 países que monitorea la OIT, solo 72 tienen regímenes de seguro de desempleo. La mayoría son países de medianos y altos ingresos... ...Más de 70 por ciento de los trabajadores en el mundo no tiene acceso al seguro de desempleo ni a ningún tipo de asistencia en caso de pérdida del empleo... ....El número de desempleados sin seguro de desempleo es aún mayor (86 por ciento) si se incluye a aquellos que no han contribuido al sistema de seguridad social el tiempo suficiente y, por lo tanto, no pueden acceder a un seguro de desempleo... (ya que) muchos regímenes de seguro se basan en las contribuciones (del trabajador y del empleador)'

Otra modalidad de atención a los cesantes es la denominada compensación por tiempo de servicios (CTS) consistente en un beneficio de carácter económico a favor del trabajador cuando este pierde o cesa en su trabajo, de manera que dicha compensación le ayuda a atender sus necesidades y las de su familia durante el período de búsqueda de un nuevo empleo. Esta compensación por tiempo de servicios, que se financia con los aportes obligatorios del trabajador mientras este está empleado, pude ser utilizada parcialmente aun cuando el trabajador no esté cesante.

Perú es el país de la región latinoamericana en la que esta modalidad de ayuda a los desempleados cesantes tuvo un desarrollo mayor. En otros países también se ha aplicado si bien con diferente denominación. En Italia se denomina "indemnización de antigüedad". En Argentina se la conoce como "indemnización por despido o antigüedad". En Bolivia se la denomina "indemnización por tiempo de servicios". En Chile, "indemnización por tiempo o años servidos". En México se la llama "indemnización de cesantía". En Ecuador se le denomina "fondo de reserva". En Colombia "auxilio de cesantía". En Venezuela "indemnización por antigüedad".

Si bien estos son los principales sistemas y modalidades actuales de protección del ingreso de las personas y de ayuda a los trabajadores cesantes, existen otros muchos de menor cobertura y con objetivos más limitados, como, por ejemplo, los fondos de emergencia social que proliferaron en la década de 1980. Sin embargo, también estos otros sistemas y modalidades difieren sustancialmente de lo que sería un fondo de estabilización del empleo. Sin embargo, aun reconociendo que existen otras modalidades de intervención, además de las aquí descritas, me he detenido las que considero las más importantes como forma de ilustrar cuál es la orientación general de las diferentes estrategias que utilizan los gobiernos en materia de mantenimiento del nivel de empleo y de los ingresos de las personas y, en algunas modalidades, los trabajadores cesantes.

9 OIT - Recomendación número 202 sobre los pisos de protección social, año 2012. 


\section{EL FONDO DE ESTABILIZACIÓN DEL EMPLEO}

Como señalé en la introducción de este artículo, existe una alternativa diferente a las modalidades de intervención descritas en la sección anterior que podría ser más eficaz que estas al generar, entre otras ventajas, menos objeciones políticas, ideológicas y económicas que las que generan propuestas como la del "trabajo garantizado" o de renta básica universal.

\section{IV.1. Caracteristicas básicas del fondo de estabilización del empleo}

Es para enfrentar el deterioro del mercado de trabajo que un fondo de estabilización del empleo (FEE) podría desempeñar, si existiera, un rol importante al actuar como un mecanismo financiado con ahorros generados durante el periodo de crecimiento del ciclo económico destinado a financiar inversiones productivas para la generación de empleo en la fase contractiva del ciclo, y, si los proyectos están adecuadamente diseñados e implementados, a contribuir a la propia recuperación de la actividad económica.

Los ahorros fiscales para constituir el FEE pueden provenir bien de ahorros del gasto corriente del Estado (es decir, ahorros del presupuesto público) bien de la aplicación de un impuesto específico y temporal a las ganancias de las grandes empresas cuando, en la fase expansiva del ciclo, esas ganancias superen promedios históricos o cuando el valor de las acciones de la empresa transadas en la Bolsa aumenta sostenida y considerablemente. En mi opinión, esta segunda fuente de recursos para alimentar el FEE (los impuestos a las ganancias en momentos de auge económico) es más adecuado que el primero debido a que no afecta a las personas físicas, especialmente de la clase media, que son las que financian la mayor parte del presupuesto público mediante impuestos indirectos como el IVA (impuesto al valor agregado) o directos como el impuesto a la renta de las personas.

Este mecanismo de financiamiento del fondo tendría una gran ventaja respecto a las propuestas que frecuentemente se hacen desde algunos círculos políticos de "poner impuestos a los ricos para ayudar a los pobres mediante inversiones sociales que, entre otras cosas, generen empleo". El problema de este tipo de propuestas es que suelen derivar en el aumento de impuestos a los ricos, pero también a la clase media; impuestos que luego se destinan en parte a financiar políticas y programas de reducción de la pobreza (con las limitaciones que estas políticas tienen, como ya señalé anteriormente) pero sobre todo a financiar un aparato burocrático sobredimensionado y, en muchos casos, ineficiente. Con el fondo de estabilización del empleo los ricos estarían realmente contribuyendo, a través de las empresas, a mejorar la situación de los más pobres (los desempleados y desempleados) en momentos de mayor necesidad social y no a reproducir ningún tipo de casta burocrática de dudosa utilidad. 
La administración del fondo podría ser estatal o, si así se considerase conveniente, tripartita, involucrando no solo al gobierno sino también a los empresarios y a los trabajadores organizados.

La operación del FEE se puede realizar mediante la ejecución bien de proyectos públicos generadores de empleo "no público" (es decir, que los beneficiarios de esos proyectos no tendrían que convertirse en funcionarios públicos "de por vida"), o mediante la asignación de fondos concursables para financiar proyectos de empleo formulados y ejecutados por el sector privado $\mathrm{y} / \mathrm{u}$ ONG, o mediante el otorgamiento al sector privado de incentivos a la contratación de trabajadores con dificultades de acceso al mercado de trabajo (jóvenes y mujeres, principalmente) o mediante cualquiera otra modalidad que asegure una utilización rápida, transparente y eficiente de los recursos del FEE. También pueden destinarse recursos del FEE a financiar proyectos de capacitación y formación profesional vinculadas a mejorar la empleabilidad de las personas sin empleo.

Una pregunta, sumamente pertinente, que se puede hacer en relación al FEE es cuál será su impacto sobre el funcionamiento del mercado de trabajo y sobre todo sobre los mecanismos de determinación del salario.

Si el FEE se utiliza, como debe ser, para generar empleo que sustituya al empleo destruido por efectos de la contracción de la actividad económica, entonces el nivel de empleo se mantendrá estable o constante sin que tenga efectos significativos sobre el consumo privado y, por tanto, sobre el nivel de precios (inflación). Tampoco debería tener mayor efecto, por las mismas razones, sobre el nivel de los salarios, salvo que el salario pagado por los programas de generación de empleo financiados por el FEE sea superior al salario de mercado, algo a lo que quienes dirijan la operación del FEE deben estar atentos para evitar que eso suceda.

El FEE sí puede tener un impacto positivo, además del de mantener el nivel de empleo previo a la recesión económica, sobre el nivel de desempleo (al generar empleo para trabajadores cesantes y evitar el aumento del desempleo) y sobre la composición del conjunto de trabajadores ocupados al evitar, en la medida de lo posible, que los trabajadores cesantes como consecuencia de la contracción de la actividad económica se auto empleen o se empleen en el sector informal, aumentando por ello el tamaño de este.

\section{IV.2. Diferencias entre el FEE y las demás modalidades de intervención}

¿El fondo de estabilización del empleo que se acaba de describir en sus características e impactos principales, es muy diferente de las modalidades de intervención descritas anteriormente o son solo una variación menor de estas?

Las similitudes entre una política de "trabajo garantizado" y un fondo de estabilización del empleo son evidentes. Sin embargo, existe una diferencia importante y es el hecho de que el FEE solo operaría en el momento contractivo del ciclo económico, mientras que la política de TG tiene una vocación de permanencia con el objetivo de mantener siempre estable el nivel de empleo y de precios. Es decir, la utilización del TG 
como mecanismo de control de la inflación es una de sus principales finalidades, lo que no ocurriría con el FEE, cuyo objetivo principal es mantener el nivel de empleo y evitar el aumento de la pobreza.

Es indudable que, en términos de lo deseable, una política de TG sería más deseable y sin duda mejor (por la amplitud de sus objetivos) que la existencia de un fondo (anticíclico) de estabilización del empleo. Sin embargo, también es indudable que la política de TG es difícilmente financiable para la mayoría de los países y su implementación enfrentaría además múltiples objeciones políticas provenientes del liberalismo económico que consideran que una política de este tipo desincentivaría el esfuerzo personal y los aumentos de productividad y de competitividad.

Por otra parte, las diferencias entre la renta básica universal y un fondo de estabilización del empleo como el descrito al inicio de este artículo son evidentes. En primer lugar, el Fondo no sería de aplicación universal sino que beneficiaría sólo a los colectivos, grupos o sectores sociales que requieren un empleo. En segundo lugar, el fondo financiaría la creación de empleo solo en épocas de retracción económica y deterioro social (aumento de la pobreza). En tercer lugar, el fondo no tendría ninguna relación impositiva con el nivel de renta o ingresos de las personas, ya que el único requisito para beneficiarse del mismo es no tener un empleo que genere ingresos, independientemente de si tiene o no algún otro ingreso (por ejemplo, alquileres) y si paga o no impuestos por cualquier concepto.

$\mathrm{Al}$ igual que en el anterior caso de la renta básica universal, las diferencias entre la renta minima de inserción y el fondo de estabilización del empleo también son muchas y muy evidentes. En primer lugar, el fondo no otorgaría prestaciones ni monetarias ni en especie ni tampoco exoneraciones de pago, sino que financiaría inversiones generadoras de empleo para beneficiar quienes estén desocupados. En segundo lugar, el fondo no estaría relacionado con el tamaño de la familia, ya que, como se ha señalado reiteradamente, su finalidad es generar empleo para el que no lo tiene, con independencia de si tiene familia o no y, en caso afirmativo, del tamaño de la misma.

También las diferencias entre un seguro de desempleo y un fondo de estabilización del empleo son notorias. Mientras que el seguro de desempleo se otorga por el derecho generado por el trabajador a percibir una prestación cuando queda cesante, el fondo no se correspondería con un derecho individual resultante de la realización de aporte alguno sino que, más bien, es un previsión de recursos financieros que el Estado hace para generar empleo en momentos de desaceleración de la economía y de caída de la tasa de ocupación.

Finalmente, las diferencias entre la compensación por tiempo de servicios, cualquiera sea su denominación, y un fondo de estabilización del empleo son muy similares a las que ya se han mencionado en el caso del seguro de desempleo.

Por todo lo anterior, las distintas propuestas de defensa de los ingresos de las personas y sus familias (renta básica universal o renta mínima de inserción) o de apoyo a 
los trabajadores cesantes en modo alguno hacen innecesaria la existencia de un fondo de estabilización del empleo, entendido este último como el ahorro y la provisión de recursos fiscales en el período de crecimiento del ciclo económico, destinados a financiar inversiones generadoras de empleo en el periodo de retracción del ciclo.

No se trataría, por tanto, de un fondo destinado a otorgar prestaciones individuales ni tampoco a asegurar una renta básica o mínima a las personas, tanto desempleadas como no. Se trata fundamentalmente de un mecanismo para garantizar inversiones productivas para la generación de empleo en periodos de contracción de la actividad económica. Se trataría de inversiones en actividades de producción, rentables y perdurables en el tiempo, de modo que no solo contribuyan a generar empleo sino que también cooperen en la recuperación de la actividad económica.

\section{INVITACIÓN AL DEBATE}

Obviamente, lo aquí señalado son solo los rasgos generales de lo que podría ser un fondo de estabilización del empleo así como de sus hipotéticas ventajas y sus diferencias con otras modalidades de intervención. Sin embargo, es evidente que también podría tener desventajas así como dificultades técnicas para su implantación en los respectivos países de la región. También es posible desarrollar una metodología de intervención para la estabilización del (nivel de) empleo que combine varias de las modalidades y enfoques presentados en este artículo.

Lo importante es que, como se señala en el título de este artículo, se impulse un debate serio y constructivo sobre este tipo de mecanismos o modalidades de intervención. Sería una forma de contribuir a enfrentar los problemas de empleo que recurrentemente enfrentan todos los países del mundo y muy particularmente los países en vías de desarrollo como son la mayoría de los países de América Latina. 